\title{
Predictive Value of Platelet-to-Lymphocyte Ratio and Systemic Immune-Inflammation Index in Adverse Pathology at Radical Prostatectomy in Patients with Low-Grade Prostate Cancer
}

jiatong zhou

Tianjin Medical University Second Hospital

ranlu liu (D16622080858@163.com )

Tianjin Medical University Second Hospital

\section{Research}

Keywords: prostate cancer, systemic immune inflammation index, neutrophil to lymphocyte ratio, adverse pathology

Posted Date: February 10th, 2021

DOl: https://doi.org/10.21203/rs.3.rs-201740/v1

License: (c) (1) This work is licensed under a Creative Commons Attribution 4.0 International License. Read Full License 


\section{Abstract}

Purpose: To determine the potential role of several biochemical and clinical markers in predicting adverse pathology (AP) and ISUP GG upgrading at radical prostatectomy (RP) with low-grade (ISUP Gleason Group (ISUP GG) 1 and 2) prostate cancer (PCa).

Methods: We retrospectively reviewed the patients who underwent radical prostatectomy following criteria: clinical stage T2a or less, and were identified low-grade PCa (ISUP GG 1-2, prostate-specifific antigen (PSA) $<20 \mathrm{ng} / \mathrm{ml}$ ) through prostate biopsy, univariate and multivariate analyses were performed to evaluate the association of patient and tumor characteristics with reclassification, AP was defined as stage $\geq T 3$ and/or ISUP GG $\geq 3$.

Results: A total of 155 patients were eligible for this study. AP at RP occurred in 20 of $97(20.62 \%)$ patients with ISUP GG 1, and 28 of 58

(48.28\%) with ISUP GG 2. At univariate analysis, bioptic ISUP GG emerged as significant factors of AP(p囚 0.001). Platelets to lymphocyte ratio(PLR) might be the risk factor of the incidence of $A P(p=0.059)$. At multivariate analysis, we found PLR and bioptic ISUP were independent significantly factors in predicting AP. The area under the curve for PLR was 0.592. And also, we showed that systemic immune inflammation index(SII) and bioptic ISUP GG were significantly associated with ISUP GG upgrading after RP in multivariate analysis.

Conclusions: We found that SII could not be a significant risk factor of AP at low-grade prostate cancer (PCa) after RP.While SII might be a predict factor for ISUP GG upgrading. PLR might be used as an independent predictor which was inversely correlated with presence of AP in low-grade PCa after RP.

\section{Background}

Inflammation plays a key role in the occurrence and development of many tumors. Inflammatory oxidative stress stimulation may cause normal cells to produce relatively abnormal protein expression and DNA damage, which is associated with the occurrence of cancer[1]. In fact, tumor-related inflammation is considered to be a key factor for tumor invasion, migration and metastasis in many cancer[2, 3]. The immune system can also recognize and eliminate transformed tumor cells that express modified antigens, a phenomenon known as immune surveillance[4]. At present, many clinical studies commonly use some inflammation indicators to predict the inflammation level.

The ratio of neutrophil to lymphocyte ratio (NLR) has been proposed as an indicator of cancer-related inflammation and poor prognosis of several types of cancer $[5,6]$. Jang et al reported that preoperative NLR could be used as an independent factor to predict cancer-specific survival[7]. Gokce et al proposed that higher NLR was related with higher Gleason Score of prostate cancer(PCa) in their study[8]. The prognostic nutritional index(PNI) and systemic immune inflammation index(SII) could demonstrated the nutritional status and inflammation level of the human body. The previous studies also showed the 
predictive value of PNI and SII in various tumors[9, 10]. However, few studies have shown the predictive role of $\mathrm{PNI}$ and SII in the prognosis of PCa. Therefore, in this present study, we invested the relationship between PNI, SII, NLR, platelets to lymphocyte ratio(PLR) and pathology results(adverse pathology, ISUP GG upgrading) in patients with low-grade PCa having undergone RP.

\section{Methods}

Patients who underwent prostate biopsy were were diagnosed with low-grade PCa (International Society of Urological Pathology grade [ISUP GG] 1 or $2, \mathrm{PSA}<20 \mathrm{ng} / \mathrm{ml}$ ) and underwent radical prostatectomy(RP) in the second hospital of Tianjin Medical University from April 2010 to May 2020. Exculsion standards were as follows: (1) patients with any other malignant tumor, (2) Patients who have received neoadjuvant therapy or 5a-reductase inhibitor therapy, (3) patient had a biopsy in another institution, (4) patients with PSA囚20 ng/ml, (5) Patients without complete clinical data.

\section{Study End Points}

The primary end points of the study were to determine the accuracy of PNI, SII, NLR, PLR in predicting AP and ISUP GG upgrading after RP. The definitions of PNI, SII, NLR, and PLR were shown as follows: PNI = albumin $(\mathrm{g} / \mathrm{L})+5 \times$ total lymphocyte counts $\left(10^{9} / \mathrm{L}\right)$; SII = platelet $\times$ neutrophil/lymphocyte counts; NLR = neutrophil/lymphocyte counts; and PLR = platelet/lymphocyte counts. Adverse pathology(AP) at RP defined as ISUP GG $\geq 3$ and/or extraprostatic disease ( $\geq T 3$ ).

\section{Statistical Analysis}

The entire statistical process was performed with SPSS 22.0 software. Non-normal and continuous variables are expressed as mean $\pm \mathrm{SD}$. The Pearson's chi-square test was used to compare dichotomous variables. Univariate and multivariate logistic regression analysis were used to screen out the independent risk factors for adverse pathology of all biopsy patients. All analyses are bilateral analysis, $p$ $<0.05$ has statistical significance.

\section{Results}

Between April 2010 to May 2020, a total of 155 patients underwent PBx and RP at our Institution. Among them, 155 patients, namely 99 (63.8\%) with ISUP GG $\leq 1$ PCa and 56 (36.2\%) with ISUP GG 2 PCa were eligible for this study. The mean age $( \pm S D)$ of the study subjects was $65.9( \pm 6.86)$ years, and the mean PSA was 9.64 ( \pm 4.32; Table 1). At multivariate analysis, we found PLR, bioptic ISUP GG kept predictive value in the incidence risk of $\mathrm{AP}$ (Table 3). The AUC of the model was 0.592 of PLR(Fig. 1). Univariate analysis showed that the incidence risk of ISUP GG upgrading (Table 2), was significantly associated with Biopsy ISUP GG( $p=0.043)$. Multivariate analysis demonstrated that SII and Biopsy ISUP GG had a significant association with ISUP GG upgrading after RP(all $p<0.05)$. 
Table 1

Characteristics of the study cohort

\begin{tabular}{|c|c|}
\hline Mean \pm SD & \\
\hline Age, years & $65.9 \pm 6.86$ \\
\hline PSA,ng/ml & $9.64 \pm 4.32$ \\
\hline BMI & $25.1 \pm 2.75$ \\
\hline PNI & $52.8 \pm 5.72$ \\
\hline SII & $566.74 \pm 390.68$ \\
\hline NLR & $2.83 \pm 2.33$ \\
\hline PLR & $127.44 \pm 50.86$ \\
\hline Biopsy ISUP & \\
\hline$\leq 1$ & $99(63.8 \%)$ \\
\hline 2 & $56(36.2 \%)$ \\
\hline Final Path Is & \\
\hline 1 & $56(36.2 \%)$ \\
\hline 2 & $71(45.8 \%)$ \\
\hline$\otimes 2$ & $28(18 \%)$ \\
\hline Pathology s & \\
\hline$\leq \mathrm{pT} 2$ & $116(75 \%)$ \\
\hline pT3 & $39(25 \%)$ \\
\hline ISUP upgrac & \\
\hline Absent & $88(56.8 \%)$ \\
\hline Present & $67(43.2 \%)$ \\
\hline Any adverse & \\
\hline Absent & 107(69\%) \\
\hline Present & $48(31 \%)$ \\
\hline
\end{tabular}


Table 2

univariable and multivariable analysis for the association between AP and patients and tumor characteristics

\begin{tabular}{|c|c|c|c|c|c|c|}
\hline \multirow[t]{2}{*}{ Variable } & \multicolumn{3}{|c|}{ Univariate } & \multicolumn{3}{|c|}{ Multivariable } \\
\hline & $\begin{array}{l}\text { Odds } \\
\text { ratio }\end{array}$ & $95 \% \mathrm{Cl}$ & $\begin{array}{l}P \\
\text { value }\end{array}$ & $\begin{array}{l}\text { Odds } \\
\text { ratio }\end{array}$ & $95 \% \mathrm{Cl}$ & P value \\
\hline Age(years) & 1.016 & $\begin{array}{l}0.966- \\
1.067\end{array}$ & 0.543 & & & \\
\hline $\mathrm{PSA}(\mathrm{ng} / \mathrm{ml})$ & 1.052 & $\begin{array}{l}0.971- \\
1.139\end{array}$ & 0.217 & & & \\
\hline BMI & 1.034 & $0.913-1.17$ & 0.602 & & & \\
\hline PNI & 1.034 & $0.972-1.100$ & 0.293 & & & \\
\hline SII & 0.999 & $0.998-1.000$ & 0.113 & & & \\
\hline NLR & 0.896 & $\begin{array}{l}0.811- \\
0.990\end{array}$ & 0.367 & & & \\
\hline PLR & 0.992 & $0.984-1.000$ & 0.059 & 0.99 & $\begin{array}{l}0.987- \\
0.994\end{array}$ & $<0.001$ \\
\hline $\begin{array}{l}\text { Biopsy ISUP GG( } \leq 1 \text { or } \\
\text { 2) }\end{array}$ & 3.593 & $\begin{array}{l}1.763- \\
7.325\end{array}$ & $<0.001$ & 2.832 & $\begin{array}{l}1.449- \\
5.498\end{array}$ & $0.002^{*}$ \\
\hline \multicolumn{7}{|c|}{$\begin{array}{l}\text { PSA prostate-specifc antigen, BMI body mass index, NLR neutrophil to lymphocyte ratio, PLR platelets } \\
\text { to lymphocyte ratio, PNI prognostic nutritional index, SIl systemic immune inflammation index, }\end{array}$} \\
\hline
\end{tabular}


Table 3

univariable and multivariable analysis for the association between ISUP GG upgrading and patients and tumor characteristics

\begin{tabular}{|c|c|c|c|c|c|c|}
\hline \multirow[t]{2}{*}{ Variable } & \multicolumn{3}{|c|}{ Univariate } & \multicolumn{3}{|c|}{ Multivariable } \\
\hline & $\begin{array}{l}\text { Odds } \\
\text { ratio }\end{array}$ & $95 \% \mathrm{Cl}$ & $P$ value & $\begin{array}{l}\text { Odds } \\
\text { ratio }\end{array}$ & $95 \% \mathrm{Cl}$ & $P$ value \\
\hline Age(years) & 1.006 & $\begin{array}{l}0.96- \\
1.054\end{array}$ & 0.802 & & & \\
\hline $\mathrm{PSA}(\mathrm{ng} / \mathrm{ml})$ & 0.998 & $\begin{array}{l}0.926- \\
1.076\end{array}$ & 0.961 & & & \\
\hline BMI & 0.998 & $\begin{array}{l}0.976- \\
1.001\end{array}$ & 0.068 & & & \\
\hline PNI & 1.006 & $\begin{array}{l}0.951- \\
1.003\end{array}$ & 0.843 & & & \\
\hline SII & 0.999 & $\begin{array}{l}0.998- \\
1.000\end{array}$ & 0.081 & 0.999 & $\begin{array}{l}0.998- \\
1.000\end{array}$ & $0.049^{*}$ \\
\hline NLR & 0.873 & $\begin{array}{l}0.721- \\
1.057\end{array}$ & 0.165 & & & \\
\hline PLR & 0.999 & $\begin{array}{l}0.993- \\
1.005\end{array}$ & 0.769 & & & \\
\hline $\begin{array}{l}\text { Biopsy ISUP GG }(\leq 1 \text { or } \\
\text { 2) }\end{array}$ & 0.497 & $\begin{array}{l}0.253- \\
0.979\end{array}$ & $0.043^{*}$ & 0.453 & $\begin{array}{l}0.227- \\
0.453\end{array}$ & $0.025^{*}$ \\
\hline \multicolumn{7}{|c|}{$\begin{array}{l}\text { PSA prostate-specifc antigen, BMI body mass index, NLR neutrophil to lymphocyte ratio, PLR platelets } \\
\text { to lymphocyte ratio, PNI prognostic nutritional index, SIl systemic immune inflammation index, }\end{array}$} \\
\hline
\end{tabular}

\section{Discussion}

Previous studies[11, 12] had shown in their studies that most patients with low-grade PCa through biopsy might have pathological upgrade/upstage after RP. Although many recent studies have found some valuable clinical markers or parameters that can predict postoperative pathological upgrade in patients with low-risk $\mathrm{PCa}[13,14]$.There were no clinically recognized biomarkers or other indicators that could determine whether low-grade PCa would upgraded after surgery.

At different stages of tumor development, the inflammatory response plays a decisive role, including initiation, promotion, malignant transformation, invasion and metastasis[15, 16].The level of inflammation in the human body has an important impact on the risk of tumor development and the prognosis of cancer patients. 
Some inflammation indicators including NLR, PLR were considered as effective predictors for predicting the prognosis of malignant tumors in some studies $[17,18]$. Ferro et al demonstrated that NLR, PLR were predictors of Gleason upgrading but not with upstaging in low-risk $\mathrm{PCa}[19]$. Gokce et al also proposed that higher GS was associated with higher NLR in patients with PCa[20].These conclusions seemed to indicate that inflammation may promote the occurrence and development of PCa. However, in our study, we found that NLR and PLR did not predict postoperative ISUP GG upgrading in patients with low-grade PCa. However, in multivariate analysis, we found that only PLR was inversely related to the risk of AP after RP. In addition to the inflammation indicators shown by blood, Sanguedolce et al reported a meaningful conclusion that low-grade inflammation of the bioptic tissue may increase the risk of postoperative AP in patients with low-grade PCa[21]. This opinion was similar to ours, the low-grade inflammation of prostate tissue may predict poor outcomes. Therefore, the role of inflammation in the occurrence and development of PCa remains controversial. Recently, some studies had found that PNI and SII were used as independent predictors to predict the prognosis of some tumors $[9,10]$. However, in our study, PNI could not play a role in predicting the risk of AP and ISUP GG upgrading in patients with low-grade PCa. However, in multivariate analysis, SIl was an independent protective factor for the risk of ISUP GG upgrading. These blood-based inflammatory factors can assess the level of preoperative inflammation in patients. The increase in tissue inflammatory cell infiltration caused by the increase of inflammatory cells in the blood may affect the growth of normal prostate tissue.

There were few limitations in our study. First, our research was a retrospective study, so some information or data may be biased. Besides, other inflammatory factors may also affect the results of the study, but we did not include these factors. Furthermore, We have included a small number of population, and the results may be biased.

\section{Conclusion}

In conclusion, PLR is an independent predictor of AP in patients with low-grade PCa after RP. And also, SII may be an important predictor of ISUP GG upgrading. However, we still need further large research to identify our results.

\section{Declarations}

Ethics approval and consent to participate

The study was approved by the Regional Ethical Review Board in Tianjin medical university second hospital. Written informed consent was obtained from all participants included in the study.

Consent for publication

Not applicable.

Availability of data and materials 
All data generated or analyzed during this study are included in this

Competing interests

The authors declare no conflict of interest.

Funding

This project was support by: Tianjin Science and technology committee(19ZXDBSY00050) and Tianjin Health Science and technology project(No.ZC20116). The founding bodies had no influence on the design of the study, data collection, data analysis, and interpretation of data; or writing of the manuscript.

Authors' contributions

Conception and Design: JZ and RL; Extraction of Data: TL and JZ;

Drafting the Article: JZ and RL; Revising It for Intellectual Content: JZ, RL; Final Approval of the Completed Article: JZ, RL. All authors read and approved the final manuscript.

Acknowledgments

We owe our thanks to Tao Li and Jiatong Zhou for their work on revising and data extracting in this manuscript.

Further information

Not applicable.

\section{References}

1. Reuter S, et al. Oxidative stress, inflammation, and cancer: How are they linked? Free Radic Biol Med. 2010;49(11):1603-16.

2. Mantovani A, et al. Cancer-related inflammation. Nature. 2008;454(7203):436-44.

3. Z W, Inflammation. and cancer. nature. 2002;420(6917):860.

4. Swann JB, Smyth MJ. Immune surveillance of tumors. Journal of Clinical Investigation. 2007;117(5):1137-46.

5. Ozmen S, et al. Neutrophil-lymphocyte ratio (NLR) and platelet-lymphocyte ratio (PLR) may be superior to $\mathrm{C}$-reactive protein (CRP) for predicting the occurrence of differentiated thyroid cancer. Endocrine Regulations. 2017;51(3):131-6.

6. Lee YS, et al., Prognostic impact of a new score using neutrophil-to-lymphocyte ratios in the serum and malignant pleural effusion in lung cancer patients. BMC Cancer, 2017. 17(1).

7. Jang WS, et al. The prognostic significance of postoperative neutrophil-to-lymphocyte ratio after radical prostatectomy for localized prostate cancer. Oncotarget. 2017;8(7):11778-87. 
8. Gokce MI, et al. Evaluation of neutrophil-to-lymphocyte ratio prior to prostate biopsy to predict biopsy histology: Results of 1836 patients. Canadian Urological Association Journal. 2015;9(11-12):761-5.

9. Chen J, et al. Systemic immune-inflammation index for predicting prognosis of colorectal cancer. World J Gastroenterol. 2017;23(34):6261.

10. Feng $Z$, et al., The preoperative prognostic nutritional index is a predictive and prognostic factor of high-grade serous ovarian cancer. BMC Cancer, 2018. 18(1).

11. Audenet F, et al. Grade Group Underestimation in Prostate Biopsy: Predictive Factors and Outcomes in Candidates for Active Surveillance. Clin Genitourin Cancer. 2017;15(6):e907-13.

12. Epstein JI, et al. Upgrading and Downgrading of Prostate Cancer from Biopsy to Radical Prostatectomy: Incidence and Predictive Factors Using the Modified Gleason Grading System and Factoring in Tertiary Grades. Eur Urol. 2012;61(5):1019-24.

13. Cary KC, Cooperberg MR. Biomarkers in prostate cancer surveillance and screening: past, present, and future. London: SAGE Publications; 2013. pp. 318-29.

14. de Cobelli $\mathrm{O}$, et al., Body mass index was associated with upstaging and upgrading in patients with low-risk prostate cancer who met the inclusion criteria for active surveillance. Urologic Oncology: Seminars and Original Investigations, 2015. 33(5): p. 201.e1-201.e8.

15. Hanahan D, Weinberg RA. Hallmarks of Cancer: The Next Generation. Cell. 2011;144(5):646-74.

16. Grivennikov SI, Greten FR, Karin M. Immunity, Inflammation, and Cancer. Cell. 2010;140(6):883-99.

17. Bagante F, et al. Neutrophil-lymphocyte and platelet-lymphocyte ratio as predictors of disease specific survival after resection of adrenocortical carcinoma. J Surg Oncol. 2015;112(2):164-72.

18. Wu Y, et al., Neutrophil-to-lymphocyte and platelet-to-lymphocyte ratios predict chemotherapy outcomes and prognosis in patients with colorectal cancer and synchronous liver metastasis. World Journal of Surgical Oncology, 2016. 14(1).

19. Ferro $M$, et al. Neutrophil, Platelets, and Eosinophil to Lymphocyte Ratios Predict Gleason Score Upgrading in Low-Risk Prostate Cancer Patients. Urol Int. 2019;102(1):43-50.

20. Gokce MI, et al. Evaluation of neutrophil-to-lymphocyte ratio prior to prostate biopsy to predict biopsy histology: Results of 1836 patients. Canadian Urological Association journal. 2015;9(11-12):E7615.

21. Sanguedolce F, et al. Bioptic intraprostatic chronic inflammation predicts adverse pathology at radical prostatectomy in patients with low-grade prostate cancer. Urologic Oncology: Seminars and Original Investigations; 2020.

\section{Figures}




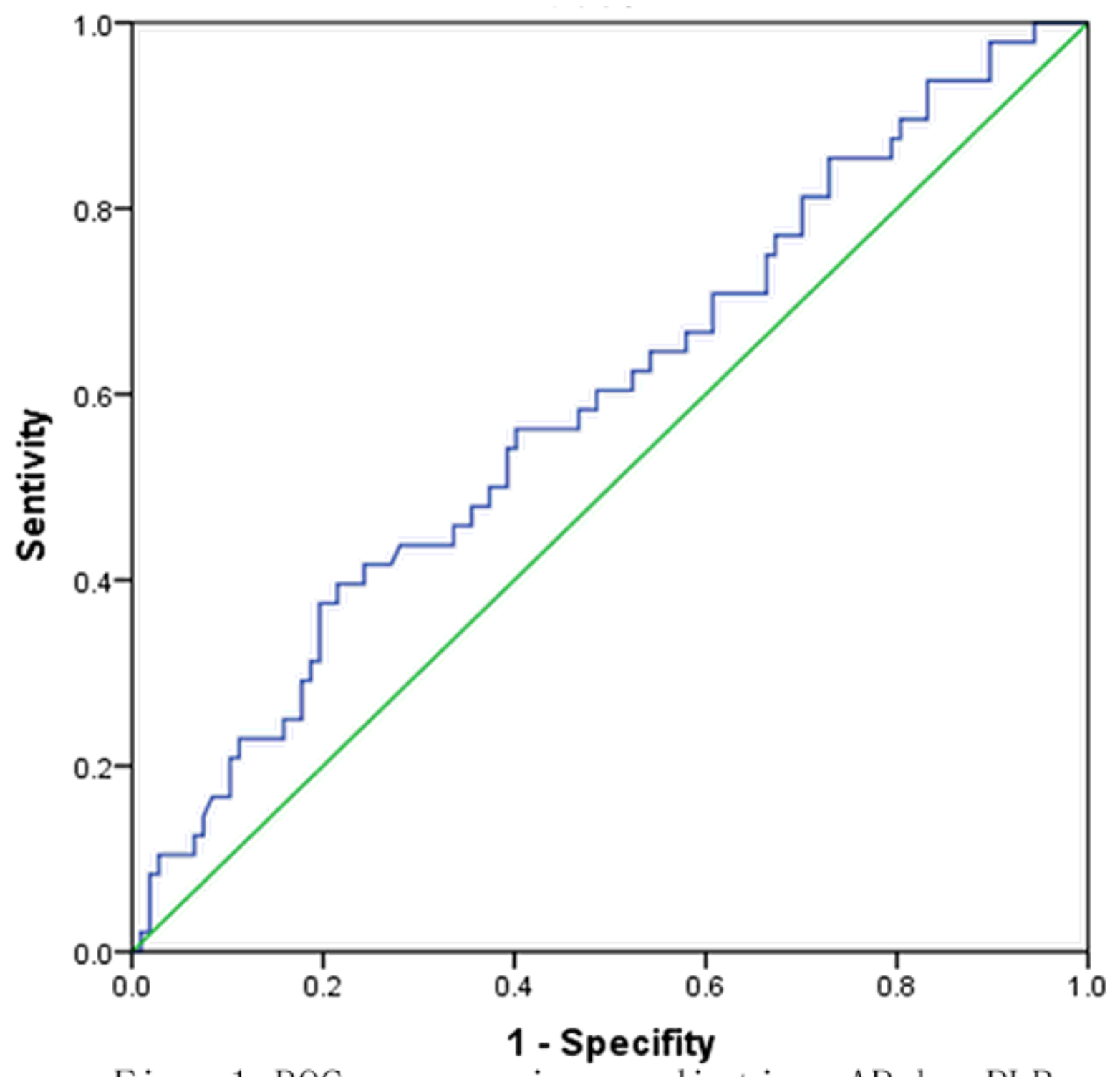

Fig. 1 ROC curves in predicting AP by PLR

Figure 1

ROC curves in predicting AP by PLR (continuous, AUC $=0.592$ ). ROC receiver operating characteristic, AUC area under curve 